\title{
Critical emancipatory reflection on a practice-based issue in relation to the working relationship between doctors and nurses in China
}

Discussion

Dong-Lan Ling ${ }^{\mathrm{a}}$, Hong-Jing Yub,"

aDepartment of Urologic Surgery, The Second Affiliated Hospital of Guangzhou Medical University, Guangzhou, Guangdong 510260, China

${ }^{b}$ Department of Nursing Administration, The Second Affiliated Hospital of Guangzhou Medical University, Guangzhou, Guangdong 510260, China

Received: 21 October 2018; Accepted: 22 December 2018; Published: 20 March 2019

Abstract: Objective: Reflection is viewed as the most significant skill and should be advocated for lifelong learning. In order to grasp the process of reflection and embrace the reflective skill, one of the authors of this paper reflects on a practice-based issue in relation to the communication between doctors and nurses, to gain new understanding and thus improve the authors' clinical practice.

Methods: Smyth's framework with four stages is utilized as an ideal framework to guide the author's reflection on the practice-based issue to free the author from her entrenched assumptions and the oppressive forces that limit her practice.

Results: The espoused and enacted theory, together with the critical reflection theory, are used to explore the values and beliefs that essentially govern the author's practice and how these are distinct from the author's worldviews. Following this, critical emancipatory reflection is undertaken to explore the dominant power structures within the author's workplace. Furthermore, given the hegemonic and chaotic working context of this issue, hegemony and symbolic interaction theory are applied to unearth the various hidden constraining and oppressive forces. Additionally, socialization theory is utilized to help the author achieve professional identity. Conclusions: Reconstructing the practice-based issue empowers the author to realize that in the future, she should act as a reflective practitioner, creating a daily habit, staying alert to practice, seeing things freshly, finding support systems, improving communication skills, conducting reflective research, and reifying reflective practice. Ultimately, the author will be sufficiently equipped to be able to transform her practice and change its outcomes.

Keywords: reflective skill $\bullet$ critical emancipatory reflection $\bullet$ Smyth's framework $\bullet$ hegemony $\bullet$ socialization theory $\bullet$ transformative practice (c) Shanxi Medical Periodical Press.

\section{Introduction}

As an experienced operational nurse in a high-grade public hospital in Southern China with $>13$ years of clinical nursing experience, I (Dong-Lan Ling) love the thrill and challenges of working on the front line, where I can spend time with patients and deliver care to them. Receiving patients' trust and compliments from time to time due to my professional dedication and clinical proficiency, I have always been proud of my career. Additionally, as a senior nurse in my health-care team, I am respected by my nurse colleagues, who often ask me to assist them in tackling complex clinical problems. However, after working routinely for years, I felt that I worked like a robot, nursing patients in a habitual way.

How to cite this article: Ling DL, Yu HJ. Critical emancipatory reflection on a practice based issue in relation to the working relationship between doctors and nurses in China. Front Nurs. 2019; 1: xx-xx. 
I was aware that there were aspects that I could have done better in practice, and I was keen to study more to develop my professional skills and thus attain professional growth and development. Therefore, I took up the opportunity provided by the hospital where I work and went abroad - to Ireland - to study for the degree of Master of Science in Nursing. Now, I have finished a 1-year course and studied the reflection module, which is quite new for me since this curriculum is not present in the nursing education syllabus in my country. After being tutored by lecturers and reading a great number of books and articles in relation to reflection, I am inspired and empowered by the knowledge that I gained from the learning process.

Reflection, which is defined as "a deliberate thinking about action with a view to improve", ${ }^{1}$ is a fundamental skill of health-care professionals and plays an important role in ensuring the quality of care in health-care practice. Its advantages have been verified in numerous studies. In order to grasp the process of reflection and embrace the reflective skill, I reflect on a practice-based issue in relation to hegemony and power relationship between doctors and nurses in the workplace to gain new understanding and thus improve the my clinical practice.

\section{Methods}

Although numerous useful reflective models have been developed in recent decades, Smyth's framework ${ }^{2}$ is one of the commonly utilized models in nursing practice, which helps to critique the status quo in power relationships and increases nurses' awareness of a sense of personal power, autonomy, and agency in their practice, eventually bringing about positive changes in their work. ${ }^{3}$ Therefore, Smyth's framework with four stages will be applied to structure the reflection on this practicebased issue. Additionally, in this article of reflection, the first person "I" is used when considering my personal and experiential knowledge, so that the "emotional and sensuous nature of the experience" is not lost as would be the case in the third-person, academic writing style. ${ }^{4}$

\section{Results}

\subsection{Describe}

I have been working in the Urology Department for $>8$ years, and normally, I am in charge of >10 postoperative patients on a daily basis. My work is fully occupied because of the overloaded intravenous transfusion prescribed for each patient, resulting in lack of time for me to interact with patients. Some of the patients could eat a normal diet, but doctors still prescribed too much nutritional intravenous fluid for them. I used to recommend doctors to reduce unnecessary transfusion treatment; however, they would refuse to do so. When I expressed my concerns to the doctors, they would respond with impatience and even label me as a troublemaker. Eventually, their tactic effectively silenced me. I felt guilty and upset as I was incapable to meet patients' needs in time. Meanwhile, I felt powerless, frustrated, and thwarted as my voice was not heard.

\subsection{Inform}

This practice-based issue is a common occurrence, and there does not appear to be enough attention given to it. ${ }^{5}$ I feel it is worthwhile to explore this issue in terms of fulfillment of patient-centered care. To unearth my involvement in this scenario, I look at my part with the eyes of an observer standing back from the action, and I realize that this situation is awkward since the nursepatient relationship is very negative in this scenario. For example, if I come across a cancer patient who is in tears, undoubtedly, I should spend a period of time just being there to support the patient's needs. However, I might have to head to another task when the call bell rings, since I need to complete so many transfusion treatments.

In this case, I realized that I was aware of my patients' psychological needs, but I did not take immediate action, which is incongruent with delivering patient-centered care ${ }^{6}$ However, staying with the patient is in conflict with task demands. These irreconcilable demands made me feel embarrassed and upset, as I believe failing to meet the patient's individualized needs is not delivering appropriate psychological care. ${ }^{7}$ Darbyshire et al. ${ }^{8}$ clearly state that "nurses should adopt the role of the sentinels of yesteryear and systematically 'watch' for occurrences of substandard care" (p. 2). Ignoring patients' feelings and letting them down are inconsistent with compassionate care and conflict with nurses' values, such as compassion and empathy, leading to care erosion. ${ }^{7}$ Additionally, it seems as though I act according to the belief that completing various tasks required by my work setting is of top priority. Nonetheless, as a nurse, I am expected to be competent to provide patientcentered care in my practice, ${ }^{6}$ which not only can enhance patients' satisfaction in terms of care but also can promote their sense of well-being and help to establish a therapeutic environment. ${ }^{9}$ I therefore agree with Priest ${ }^{10}$ that, as a nurse, I should provide "expressive care" for patients rather than "instrumental care". Hence, it is my responsibility to recognize patients' needs and assess the risk to fulfill the desired healthcare outcomes. I felt guilty not being able to spend time interacting with and comforting the patients as needed. 
Furthermore, I believe it is my obligation to advocate patients' interests and express my concerns to doctors. ${ }^{11}$ Overuse of intravenous infusions may put patients in danger, lead to patients losing their appetite, and cause unnecessary financial burden. 5,12 However, because of the doctor-nurse hierarchy, I found that there is no avenue for me to express my concerns for my patients. Since teamwork and collaboration are essential to ensure high-quality health-care service delivery, ${ }^{13,14}$ I put much value on interprofessional collaboration within my health-care team. Nevertheless, I found that there are few opportunities of utilizing my specialism to contribute to the patient care plan. I routinely scrutinize and execute medical prescriptions and communicate with doctors if I have any query about the prescriptions. I always respect them and believe that we should have mutual respect. However, I am not responded to in the same way and my concerns about patient safety are not heard and respected. I hoped to collaborate more with doctors, ${ }^{14}$ but I felt like I was just a robot that implements their orders since no one would value my voice. Sometimes, patients complained about the endless intravenous infusion to me and I wanted to question doctors' decisions, but I did not because I worried that if I insisted on questioning their orders, it might seem as if I were challenging their knowledge. I also feared that my opinions were not the common beliefs within the team, so they would perceive me as an eccentric and antipathetic colleague. I felt it was pointless to discuss and argue with them anyway. Therefore, my self-efficacy was constrained strongly by these powerless feelings.

\subsection{Confront}

After informing my practice-based issue, it is evident that there are contradictions between my espoused and enacted worldviews, which have been in conflict within me for years. Schön ${ }^{15}$ pointed out that there is a gap between espoused and enacted values for many professionals. As indicated by Fook and Gardner, ${ }^{16}$ the most effective way of change is to explore and learn what worldviews actually rule your practice and how they are different from your espoused theories. This could be achieved by using the critical reflection theory, which integrates analyses of personal values and beliefs regarding the way power is exercised and the impacts of socially dominant thinking accordingly. ${ }^{17,18}$ Briefly, critical reflection would allow me to unearth my hidden assumptions, which in turn would help me to bring about practice changes. ${ }^{19,20}$

From my story, I can see that my espoused values and beliefs are respect, collaboration, compassion, and equality. Historically, these values are rooted in my large family, which has a strong belief in these values, requiring cooperation with and caring for one another. In addition, my 17 years of schooling and 10 years of work experience also have a significant impact on me in upholding these values, leading me to believe that as a senior nurse, I should respect and collaborate with coworkers. As stated by Shahriari et al., ${ }^{21}$ compassion and collaboration are the key values of a nursing professional; thus, I bear this in mind and try to uphold and embody these values in my working relationships with doctors. Similarly, I hope doctors will treat me in the same way and communicate with me in a professional and respectful way, as well as value my knowledge and skills. ${ }^{14}$ Yet, this scenario illustrates that I am not an equal member of the team and I am powerless because of the doctor-nurse hegemonic structure, which is at odds with my "espoused theory". ${ }^{15}$ Consequently, I am not always able to maintain my rights for my espoused values to be reciprocated in the face of enacted values.

It is reported that teamwork in the health-care system is essential to ensure high-quality health-care service delivery, ${ }^{14,22}$ but if medical professionals in the team do not value equality and collaboration, then these values cannot be exercised. ${ }^{3}$ Interprofessional collaboration only exists where practitioners are willing to trust coworkers and recognize their knowledge and skills. ${ }^{22,23}$ However, in my story, doctors utilized their power to exercise superiority over me. My willingness to establish rapport and share these values with them was inspiring, but the reality was far from these ideals. Evidently, effective interprofessional collaboration has not filtered down in my workplace. I believe that values including respect, collaboration, and equity should serve all stakeholders' interests in the multidisciplinary teams within hospitals, since it is beneficial for all of them, especially for patients. ${ }^{24}$ It is imaginable that if every professional in the hierarchy endorses these values, then everyone's awareness will be in check, and my job satisfaction will be much higher.

When I explore tensions between the doctors and me, it becomes necessary to classify theories related to power. Modernists believe that power is owned by someone who is at a higher position in the social structure, while postmodernists view power as knowledge. ${ }^{16}$ To explore the power relations and constraints that are involved in this scenario, critical emancipatory theory, which is based on the work of Smyth ${ }^{3}$ and Street, ${ }^{25}$ is selected. This theory will help me to critique the status quo in the power relationship and increase my awareness of a sense of personal autonomy, and ultimately, will bring about a positive change in practice. ${ }^{3,19}$ Essentially, I found that my sense of powerlessness could be related to various constraints. 
The cultural constraint has deeply influenced my actions in practice. Specifically, China is an ancient country and has been deeply affected by Confucianism for $>2000$ years. The Confucian culture is characterized by a strong legacy of patriarchy ${ }^{26}$; consequently, paternalism is prevalent within Chinese organizations. ${ }^{27}$ It is unsurprising to see that doctors exercise paternalism within organizations. ${ }^{28,29}$ Hegemony, which refers to the demand for undisputable compliance from subordinates, is perceived as one of the significant characteristics of paternalism and is prevalent within organizations; as a result, subordinates' autonomy is not highly valued. ${ }^{28}$ Likewise, a doctor-dominated culture has been formed in health-care settings, and doctors have exercised a disproportionate degree of power and hegemony over nurses for a long time, resulting in a tradition of nurses being subservient to doctors. ${ }^{29}$

Moreover, due to the pervasiveness of patriarchal culture in Chinese societies, the notion of masculinity is entrenched. ${ }^{26}$ In the health-care system, males have a vested interest as they dominate medicine. ${ }^{30}$ Doctors' professional knowledge is acknowledged and valued, while the nursing profession is undervalued; ${ }^{31}$ i.e., nurses' (women's) work is generally less appreciated than doctors' (men's) work. ${ }^{32}$ Hence, doctors have enjoyed plenty of professional power in the hospital hierarchy, and the nursing profession has culturally compromised its professional standing. ${ }^{33}$

The historical constraint also affects my responses in communication with doctors. Although the health service has been nationally reformed and calls for developing a climate of collaboration, team building, and equality within hospitals, ${ }^{30}$ this situation has not changed substantially over the decades. ${ }^{22,33}$ Molleman et al. ${ }^{34}$ demonstrate that doctors commonly hold a strong view that collaboration might weaken their power over their work; thus, doctors endeavor to maintain the status quo to protect their privileges. Unfortunately, health-care systems have been tolerating ineffective interprofessional collaboration, and measures have not been taken to improve these unacceptable conditions, ${ }^{35}$ although Coombs and Ersser ${ }^{22}$ and Baggs et al. ${ }^{36}$ argue that this medical hegemony continues to impede the nurses' contribution in decision-making processes. This is proven very well in my story, when the doctors' prescriptions were not allowed to be questioned and challenged. Now, I realize that my interactions with coworkers have been deeply influenced by this historical constraint.

The social constraints can be identified by applying the symbolic interaction theory, which allows individuals to understand how they interact with people or professionals within the hierarchy. ${ }^{37}$ Since doctors have a higher social class, receive higher salaries than me, live in luxury apartments, and maintain their own social circle, I believe that they do not treat me as their social equal. All these aspects serve as a social constraint to my practice in my workplace, where there is grandstanding about the equal contributions of members.

Concerning the political constraint, it is essential to understand that the vivacity of politics is deeply rooted in the structure of modern health-care organizations and is about the power plays engaged in by individuals. ${ }^{38}$ It is particularly hard to achieve any sort of equality owing to bureaucratic and top-down supervision and control. ${ }^{39}$ We cannot disagree that organizational support plays a greater role in establishing and sustaining healthy work environments than individual attributes; ${ }^{38}$ therefore, the urgent need for bringing in change should not be constantly neglected by the health-care system. Unfortunately, we are still wearing nurse caps, which - in my opinion - is a form of power play within my organization.

Economically, health-care systems in China have been suffering from shortage of nurses for a long time due to many reasons, such as lack of investments in nursing education, high turnover rate among nursing staff, and so on. ${ }^{40,41}$ From the story described herein, it can be seen that I was in charge of $>10$ patients on a daily basis, resulting in a lack of time to care for patients. Indeed, it is challenging for organizations to balance staff sufficiency and budget savings in the current economic situation. If these situations are not paid enough attention, holistic care delivery and patient safety could be threatened as overloaded nurses would lose a sense of agency, treat patients as objects rather than people, and often overemphasize the importance of getting the work done. , $^{-42-44}$ Consequently, patients' communication needs may be ignored or even treated as an irritant ${ }^{44}$; so sufficient nursing staff are needed to enable me to provide holistic patient care in a flexible and individualized manner.

Personal constraint should, however, not be neglected. I am an introverted person by nature, feeling shy and anxious to speak in front of people. Additionally, in the medical environment - where medicine is an everchanging science undergoing continual development, ${ }^{45}$ I must face the fact that my knowledge is inadequate and outdated over the past years, and I have become unconfident in my current professional role. Moreover, a lack of communication skills also contributes to my confidence deficit.

\subsection{Reconstruct}

By confronting all these constraints in relation to my practice issue, I enter the final stage, namely, 
"reconstructing", as described in Smyth's model. Now, I realize my work contexts were so complicated that I was not the only determinant of the outcome. I ultimately understand that apart from my personal inability to make the situation better, many other factors can also contribute to this unsatisfactory outcome. According to the socialization theory, a professional identity could be internalized and developed via the acquisition of knowledge, skills, and ethical standards so that a professional role could be achieved, ${ }^{46}$ and the achievement of effective social change must depend on individual change. ${ }^{16}$ Thus, it becomes especially important for me to equip myself with knowledge so that I can alter the doctors' view that nurses are maidservants and know nothing. To remove personal constraints and gain confidence to fulfill my professional role, I must upgrade my knowledge and skills (especially, the communication skill) constantly. Furthermore, I need to create a lifelong learning habit to promote both personal and professional development. ${ }^{47}$

Communication and dialogue between doctors and myself are important so that new shared understandings can be created. ${ }^{16}$ Taylor $^{3}$ emphasizes that by setting a research group within organizations, the combination of reflective processes and action research can assist in creating effective communication and collaboration between coworkers. This ultimately facilitates the process of raising critical awareness of practice problems by the group members and uncovers constraints. Currently, I have finished my study in Ireland for the degree of Master of Science in Nursing and have gained the confidence and competence to conduct research in the future. A qualitative reflective research approach is an ideal way to bring about changes in practice since awareness, new insight, and potential for improvement could be produced by the research. ${ }^{3}$

\section{Discussion}

Reflection is considered the most important part of the learning process. ${ }^{15}$ As Dewey ${ }^{48}$ says, "We do not learn from experience... we learn from reflecting on experience" (p. 78). Contemporary nurses have always been encouraged to engage in reflective practice. Reflective practice is the process of self-examination of everyday practice so as to gain new insights into self or practice, enabling practitioners to engage in a process of continuous learning. ${ }^{22}$ Numerous studies have verified that exclusively relying on the paradigm of technical rationality and competence-based practice is not enough to deliver holistic and safe patient care. ${ }^{49,50}$ Following through the critical emancipatory reflection on my practice-based issue, I now began to recognize and understand my own values, feelings, assumptions, and behaviors that have been influenced by the power structures. By informing and conforming to the issue, I understand the interrelation between my personal constraints and work performance. Moreover, I realize that I do have the power to help, rather than doing nothing for patients. I also realize that I need to express my concerns to my coworkers in a more professional and confident manner so that they will accept my opinions. In order to address the issue, firstly, I need to promote and maintain my moral stance and agency, as well as ensure provision of physical needs and sympathetic presence to patients, to prevent my care erosion. ${ }^{7,51}$ Secondly, I should hold to the strong value of promoting patient care and must take on the responsibility to challenge assumptions, even though this may make me unpopular and difficult to live with. Thirdly, when doctors make bedside rounds for patients, I should participate in the rounds and share my knowledge with them to show my intent to collaborate and to serve patients' interests. ${ }^{52}$ Lastly, I have to endeavor to create time and space for patients by rearranging the order of my work in my future practice and making myself more efficient so that I have time to meet patients' emotional needs. "Value-based practice" should be borne in my mind to achieve patient-centered care rather than "outcomedriven" practice. ${ }^{16}$ Additionally, apart from my personal development, it is also vital for my workplace to employ sufficient nurses to liberate me from the shackles of routines and allow me to deliver patient care in a flexible and individualized style. ${ }^{53}$

\section{Conclusions}

Overall, the reflective process has enabled me to become more aware of my role in providing support for patients and has encouraged me to take action when patients appear to need help and advice. Most importantly, it has helped me to become more confident and have more inner power when confronting doctors. Reconstructing my issue empowers me sufficiently to realize that, in the future, I should act as a reflective practitioner, creating a daily habit, staying alert to practice, seeing things freshly, finding support systems, improving communication skills, conducting reflective research, and reifying reflective practice. ${ }^{3}$ Ultimately, I will be sufficiently equipped to be able to transform my practice and change its outcomes.

\section{Conflicts of interest}

All contributing authors declare no conflicts of interest. 
1. Hatton N, Smith D. Reflection in teacher education: towards definition and implementation. Teaching and Teacher Educ. 1995;11: 33-49.

2. Smyth J. Developing and sustaining critical reflection in teacher education. J Teacher Educ. 1989;40:2-9.

3. Taylor B. Reflective practice for healthcare professionals: a practical guide. $3^{\text {rd }}$ ed. Berkshire: McGraw-Hill Education. 2010.

4. Duke S. Continuing the journey with reflection. In: Butman C, Schutz S, eds. Reflective Practice in Nursing. 4th ed. Blackwell: Oxford. 2008:195.

5. Yuan S. China should reduce the overuse of intravenous infusion. BMJ. 2014;348. http://search.proquest.com/openview/076abaceb617023170fd211 4af6a197e/1 ?pq-origsite=gscholar $\&$ cbl=2040978. Accessed May 11, 2018.

6. Health Service Executive. Guiding framework for the implementation of nursing and midwifery quality care-metrics in the health service executive Ireland. Ireland: Health Service Excusive. http://www. hse.ie/eng/about/Who/ONMSD/news/GFQCM.pdf. Accessed May 31, 2018.

7. de Vries J, Timmins F. Care erosion in hospitals: problems in reflective nursing practice and the role of cognitive dissonance. Nurse Educ Today. 2016; 38:5-8.

8. Darbyshire P, Ralph N, Caudle H. Editorial: nursing's mandate to redefine the sentinel event. J Clin Nurs. 2015;24:1445-1446.

9. McCance T, McCormack B, Dewing J. An exploration of person-centredness in practice. Online $J$ Issues Nurs. http://dx.doi.org/10.3912/OJIN.Vol16No02Man01. Accessed May 25, 2018.

10. Priest $\mathrm{H}$. Effective psychological care for physically ill patients in hospital. Nurs Standard. 2010;24:48-56.

11. Somerville D, Keeling J. A practical approach to promote reflective practice within nursing. Nurs Times. 2004;100:42.

12. Chen M, Wang L, Chen W, Zhang L, Jiang $H$, Mao $W$. Does economic incentive matter for rational use of medicine? China's experience from the essential medicines program. Pharmacoeconomics. 2014; 32:245-255.

13. Rashid Al-Abri MD. Managing change in healthcare. Oman Med J. 2007; 22:9-10.

14. Zwarenstein M, Goldman J, Reeves S. Interprofessional collaboration: effects of practice-based interventions on professional practice and healthcare outcomes. Cochrane Database Syst Rev. 2009;3:CD000072.
15. Schön DA. Educating the reflective practitioner. San Francisco: Josseybass. 1995.

16. Fook J, Gardner F. Practicing critical reflection: a resource handbook. Berkshire: McGraw-Hill Education. 2007.

17. Fook J. Reflective practice and critical reflection. In: Lishman J, ed. Handbook for practice learning in social work and social care. Basingstoke: Athenaeum Press. 2007:363-375.

18. White S, Fook J, Gardner F. Critical reflection in health and social care. Berkshire: McGraw-Hill Education. 2006.

19. Jasper M. Beginning reflective practice. Cheltenham: Nelson Thornes. 2003.

20. Fook J, Askeland GA. The "critical" in critical reflection. In: White S, Fook J, Gardner F, eds. Critical reflection in health and welfare. Maidenhead: Open University Press. 2006:40-53.

21. Shahriari M, Mohammadi E, Abbaszadeh A, Bahrami M. Nursing ethical values and definitions: a literature review. Iran $J$ Nurs Midwifery Res. 2013;18:1-8.

22. Coombs M, Ersser SJ. Medical hegemony in decision-making - a barrier to interdisciplinary working in intensive care? J Adv Nurs. 2004;46: 245-252.

23. Freeman M, Miller C, Ross $N$. The impact of individual philosophies of teamwork on multi-professional practice and the implications for education. J Interprofessional Care. 2000;14:237-247.

24. Barine AK, Minja D. Transformational corporate leadership. Wask Forest, NC: Integrity Publisher. 2012.

25. Street $A$. From image to action: reflection in nursing practice. Deakin University. 1991.

26. Zhou H, Long LR. Combined morality and authoritarian and applied benevolence to convince followers: a review of paternalistic leadership. Adv Psych Sci. 2005;13:227-238 (in Chinese).

27. Ma L, Tsui AS. Traditional Chinese philosophies and contemporary leadership. Leadership Quarterly. 2015;26:13-24.

28. Xiao MZ, Wu XH. Chinese leadership: culture and confucianism. Public Integrity. 2014;16:165-172.

29. Qian WQ, Gao YY. The "Three Roles" of leaders in hospital culture construction. JiangSu Health Care Manag. 2015; 26:71-72 (in Chinese).

30. Whitehead D, Davis P. The issue of medical dominance (hegemony). J Orthopaedic Nurs. 2001;5:114-115.

31. Zelek B, Phillips SP. Gender and power: nurses and doctors in Canada. Int J Equity Health. 2003;2:1. 
32. Coburn D. Medical dominance then and now: critical reflections. Health Sociol Rev. 2006;15:432-443.

33. Gair G, Hartery T. Medical dominance in multidisciplinary teamwork: a case study of discharge decision-making in a geriatric assessment unit. J Nurs Manag. 2001;9:3-11.

34. Molleman E, Broekhuis M, Stoffels R, Jaspers F. How health care complexity leads to cooperation and affects the autonomy of health care professionals. Health Care Anal. 2008;16:329-341.

35. Moss M, Good VS, Gozal D, Kleinpell R, Sessler CN. An official Critical Care Societies Collaborative statement-burnout syndrome in critical care health-care professionals: a call for action. Chest. 2016;150:17-26.

36. Baggs JG, Norton SA, Schmitt MH, Dombeck MT, Sellers CR, Quinn JR. Intensive care unit cultures and end-of-life decision making. J Crit Care. 2007;22: 159-168.

37. Blumer H. Symbolic interactionism: perspective and method. Oakland: University of California Press. 1986.

38. Bleakley A. Working in "teams" in an era of "liquid" healthcare: what is the use of theory? J Interprofessional Care. 2013;27:18-26.

39. Bassett S, Westmore K. How nurse leaders can foster a climate of good governance: in the first of a four-part series on corporate responsibility, Sally Bassett and Kathryn Westmore explore the importance of workplace culture and leadership. Nurs Manag. 2012;19:22-24.

40. Sheer B, Wong FKY. The development of advanced nursing practice globally. I Nurs Scholarship. 2008;40:204-211.

41. Yun H, Jie S, Anli J. Nursing shortage in China: state, causes, and strategy. Nurs Outlook. 2010;58:122-128.

42. Pelzang R. Time to learn: understanding patientcentred care. Br J Nurs. 2010;19:912.
43. Zolnierek C. The importance of knowing the patient. Issues Ment Health Nurs. 2011;32:392-393.

44. Bach S, Grant A. Communication and interpersonal skills in nursing. $3^{\text {rd }}$ ed. London: Open University in assoc. with Sage. 2015.

45. Tachimori $\mathrm{Y}$, Iwanaga $\mathrm{H}$, Tahara $\mathrm{T}$. The networks from medical knowledge and clinical practice have small-world, scale-free, and hierarchical features. Physica A. 2013;392:6084-6089.

46. Young MN, Peng MW, Ahlstrom D, Bruton GD, Jiang $Y$. Corporate governance in emerging economies: a review of the principal-principal perspective. J Manag Studies. 2008;45:196-220.

47. McCormack B, Dewing J, McCance T. Developing person-centred care: addressing contextual challenges through practice development. Online $J$ Issues Nurs. http://dx.doi.org/10.3912/OJIN.Vol16No02Man03. Accessed May 31, 2018.

48. Dewey J. How we think: a restatement of the relation of reflective thinking to the educative process. Boston: D C Heath \& Co. 1933.

49. Forneris SG, Peden-McAlpine CJ. Contextual learning: a reflective learning intervention for nursing education. Int J Nurs Educ Scholarship. 2006;3:1254.

50. Epp S. The value of reflective journaling in undergraduate nursing education: a literature review. Int J Nurs Studies. 2008;45:1379-1388.

51. Pope JE. Complications of spinal cord stimulation. Reducing risks and complications of interventional pain procedures. Philadelphia: Saunders. 2012;5:3-10.

52. Manias E, Street A. Nurse-doctor interactions during critical care ward rounds. J Clin Nurs. 2001;10:442-450.

53. Kelly J. Barriers to achieving patient-centered care in Ireland. Dimens Crit Care Nurs. 2007;26:29-34. 